Research Article

\title{
Positive Recurrence and Extinction of Hybrid Stochastic SIRS Model with Nonlinear Incidence Rate
}

\author{
Liang Chen $\mathbb{D}^{1}$ and Shan Wang $\mathbb{D}^{2}$ \\ ${ }^{1}$ College of Science, Jiujiang University, Jiujiang 332000, China \\ ${ }^{2}$ Department of Mathematics, Pingxiang University, Pingxiang 337000, China \\ Correspondence should be addressed to Shan Wang; geminiws@126.com
}

Received 28 December 2020; Revised 15 February 2021; Accepted 20 February 2021; Published 3 March 2021

Academic Editor: Niansheng Tang

Copyright ( 2021 Liang Chen and Shan Wang. This is an open access article distributed under the Creative Commons Attribution License, which permits unrestricted use, distribution, and reproduction in any medium, provided the original work is properly cited.

In this paper, the dynamic behavior of a class of hybrid SIRS model with nonlinear incidence is studied. Firstly, we provide the condition under which the positive recurrence exists and then give the threshold $R_{0}^{S}$ for disease extinction, that is, when $R_{0}^{S}<1$, the disease will die out. Finally, some examples are constructed to verify the conclusion.

\section{Introduction}

Disease is one of the most dangerous enemies of human beings. COVID-19 has spread throughout the world since the end of 2019 and brought great harm to people's life and global economy. It is one of the important measures to understand the characteristics of disease transmission and control the disease to establish and study the disease model. Because of its importance and rich research content, different scholars have used various methods to study dynamical behavior and properties of epidemic models, such as persistence and extinction [1-7], stationary distribution and ergodicity $[5,8-12]$, stability $[13,14]$, bifurcation $[15,16]$, and optimal control of disease [17]. In [17], the optimal vaccination strategies were used to minimize the numbers of the susceptible and infected individuals as well as to maximize recovered individuals.

Incidence rate plays an important role in the spread of diseases. Liu et al. [18] abandoned the assumption that incidence rate is proportional to the quantity of infected and susceptible ones to study the influence of nonlinear incidence function on SIRS model. This is more reasonable and suitable for practical situations, because the premise of bilinear incidence rate is that the population is homogeneously mixed, and the probability of being infected for each person is the same. Meng et al. [3] discussed SIS model with the form $(S I /(a+I))$ and double epidemic hypothesis. In [6], Cai et al. studied the ratio-dependent transmission rate with the form $g(S, I)=\left(S^{h} I /\left(S^{h}+\alpha H^{h}\right)\right)$ in SIRS epidemic model. Recently, Wei-Xue studied the asymptotic stability of SEIR model with incidence rate $g(I) S=\left(S I /\left(1+a I^{q}\right)\right)[19]$. In this paper, we concentrate on general incidence rate with this form.

Life is full of stochastic disturbances, which often have an important impact on the system and will essentially change the properties of the system. White noise depicted by Brownian motion and telegraph noise characterized by continuous time Markov chain are two important types of noise. Some scholars have studied the effect of Levy noise on epidemic models [20, 21]. Although many scholars have studied the different properties of epidemic models with white noise and Markovian switching [22-26], there are few studies on the positive recurrence and extinction of SIRS models with the abovementioned general incidence rate. In this paper, we will study the abovementioned properties of stochastic hybrid SIRS model with the following form: 


$$
\left\{\begin{array}{l}
\mathrm{d} S(t)=\left(\Lambda\left(\alpha_{t}\right)-d\left(\alpha_{t}\right) S(t)-\frac{\beta\left(\alpha_{t}\right) S(t) I(t)}{1+a\left(\alpha_{t}\right) I^{q}}+\delta\left(\alpha_{t}\right) R(t)\right) \mathrm{d} t+\sigma_{1}\left(\alpha_{t}\right) S(t) \mathrm{d} B_{1}(t), \\
\mathrm{d} I(t)=\left(\frac{\beta\left(\alpha_{t}\right) S(t) I(t)}{1+a\left(\alpha_{t}\right) I^{q}}-\left[\mathrm{d}\left(\alpha_{t}\right)+\gamma\left(\alpha_{t}\right)+\mu\left(\alpha_{t}\right)\right] I(t)\right) \mathrm{d} t+\sigma_{2}\left(\alpha_{t}\right) I(t) \mathrm{d} B_{2}(t), \\
\mathrm{d} R(t)=\left(\gamma\left(\alpha_{t}\right) I(t)-\left[d\left(\alpha_{t}\right)+\delta\left(\alpha_{t}\right)\right] R(t)\right) \mathrm{d} t+\sigma_{3}\left(\alpha_{t}\right) R(t) \mathrm{d} B_{3}(t),
\end{array}\right.
$$

where $0<q \leq 1 . S(t), I(t)$, and $R(t)$ denote the numbers of the susceptible, infected, and recovered. $\Lambda$ is the recruitment rate, $d$ represents the natural death rate, $\beta$ means valid contact rate, $\mu$ stands for the disease-caused death rate, $\gamma$ signifies the recovery rate of infected individuals, and $\delta$ expresses the rate at which the recovered ones lose their immunity and go back to the susceptible class.

$B_{i}(t), i=1,2,3$ are independent Brownian motions, $\sigma_{i}(k), i=1,2,3$ are the intensities of the disturbances and Markov chain $\left\{\alpha_{t}\right\}_{t \geq 0}$ will be introduced in detail in Section 2. Moreover, Section 2 will give an important lemma which will be used in this paper. Obviously, Markovian switching and nonlinear incidence rate make the model more applicable, but also increase the difficulty of research. Section 3 is devoted to prove the positive recurrence and stationary distribution of model (1) by constructing suitable Lyapunov functions. Section 4 gives the threshold for disease extinction. Some examples and their simulations will be presented in Section 5 to verify our results.

\section{Preliminaries}

Some background knowledge about differential equations and an important lemma of the paper will be introduced in this section.

Throughout this paper, denote by $\left(\Omega, \mathscr{F},\left\{\mathscr{F}_{t}\right\}_{t \geq 0}, \mathbb{P}\right)$ the complete probability space with a filtration which satisfies the usual conditions. Furthermore, let $\mathbb{R}_{+}^{n}=\left\{x \in \mathbb{R}^{n}: x_{i}>\right.$ $0,1 \leq i \leq n\}$ and assume that $B_{i}(t), i=1,2,3$ are independent Brownian motions on the probability space.

Assume that $\left\{\alpha_{t}\right\}_{t \geq 0}$ is a right continuous Markov chain in a finite state space $\mathcal{N}=\{1,2, \ldots, N\}$ and its generator is $\Gamma=\left(\gamma_{i j}\right)_{N \times N}$, satisfying

$$
\mathbb{P}\left(\alpha_{t+\Delta}=j \mid \alpha_{t}=i\right)= \begin{cases}\gamma_{i j} \Delta+o(\Delta), & \text { if } i \neq j, \\ 1+\gamma_{i i} \Delta+o(\Delta), & \text { if } i=j,\end{cases}
$$

for $\Delta \downarrow 0$. Here, $\gamma_{i j}>0$ if $i \neq j$ and $\sum_{j=1}^{N} \gamma_{i j}=0$, for all $i \in \mathcal{N}$. In this paper, we assume that the Markov chain is irreducible; thus, it has a unique stationary distribution $\pi=\left(\pi_{1}, \pi_{1}, \ldots\right.$, $\pi_{N}$ ) which can be obtained by solving the equation

$$
\left\{\begin{array}{l}
\pi \Gamma=0, \\
\sum_{j=1}^{N} \pi_{j}=1 .
\end{array}\right.
$$

Considering the $n$-dimensional SDEs with Markovian switching,

$$
\mathrm{d} X(t)=f\left(X(t), \alpha_{t}\right) \mathrm{d} t+g\left(X(t), \alpha_{t}\right) \mathrm{d} B(t),
$$

with the initial value $X\left(t_{0}\right)=x_{0} \in \mathbb{R}^{n}$ and $\alpha_{t_{0}}=i_{0}$, where $f(\cdot, \cdot): \mathbb{R}^{n} \times \mathcal{N} \longrightarrow \mathbb{R}^{n}, \quad g(\cdot, \cdot): \mathbb{R}^{n} \times \mathcal{N} \longrightarrow \mathbb{R}^{n \times n}, \quad$ and $\sigma(x, k)=g(x, k) g^{T}(x, k)=\left(\sigma_{i j}(x, k)\right)$. Let $\quad C^{2,1}\left(\mathbb{R}^{n} \times\right.$ $\left.\mathcal{N} ; \mathbb{R}_{+}\right)$be the set of nonnegative functions $V(X, i)$ defined on $\mathbb{R}^{n} \times \mathscr{N}$ and the functions are continuously twice differential in $X$. The differential operator $\mathscr{L}$ on the function $V(x, i)$ is defined by

$$
\begin{aligned}
\mathscr{L} V(x, i)= & \sum_{j=1}^{n} f_{j}(x, i) \frac{\partial V(x, i)}{\partial x_{j}} \\
& +\frac{1}{2} \sum_{j=1}^{n} \sum_{k=1}^{n} \sigma_{k j}(x, i) \frac{\partial^{2} V(x, i)}{\partial x_{j} \partial x_{k}} \\
& +\sum_{j=1}^{n} \gamma_{i j} V(x, j) .
\end{aligned}
$$

Lemma 1 (see [27]). Supposing that the following three conditions hold true,

(A1) $\gamma_{i j}>0$ if $i \neq j$;

(A2) For every $i \in \mathcal{N}$ and the positive constant $\lambda, \sigma(x, i)$ satisfies $(\sigma(x, i) \xi, \xi) \geq \lambda|\xi|^{2}$

(A3) There exists a bounded set $\mathcal{U}$ with compact closure and a nonnegative function $V(x, i)$ such that $\mathscr{L} V<-\kappa$ for any $x \in \mathscr{U}^{c}$ and $i \in \mathcal{N}$ where $\kappa$ is a positive constant, and then $\left(X(t), \alpha_{t}\right)$ is the positive recurrent. That is to say, there exists a unique stationary distribution $\Pi(\cdot, \cdot)$ such that

$$
\mathbb{P}\left\{\lim _{t \rightarrow \infty} \frac{1}{t} \int_{0}^{t} h\left(X(t), \alpha_{t}\right) \mathrm{d} t=\sum_{i=1}^{N} \int_{\mathbb{R}^{n}} h(x, i) \Pi(\mathrm{d} x, i)\right\}=1,
$$

for any integrable function $h(x, i)$ with respect to $\Pi$.

For the model we discuss, our first concern is whether it has a unique positive solution. The following theorem will answer this question.

Theorem 1. For the initial value $\left(S(0), I(0), R(0), i_{0}\right) \in$ $\mathbb{R}_{+}^{3} \times \mathcal{N}$, model (1) has a unique solution $(S(t), I(t), R(t))$ and the solution is still in $R_{+}^{3}$ with probability one.

The proof is common; we omit it here. 


\section{Positive Recurrence of Model (1)}

In this section, positive recurrence of model (1) will be discussed by utilizing the theory put forward by Khasminskii under some conditions.
Theorem 2. For any initial value $\left(S(t), I(t), R(t), \alpha_{0}\right) \in$ $\mathbb{R}_{+}^{3} \times \mathcal{N}$, if

$$
R_{1}^{S}=\frac{\left(\sum_{k=1}^{N} \pi_{k}[\Lambda(k) \beta(k)]^{(1 / 3)}\right)^{3}}{\left(\sum_{k=1}^{N} \pi_{k}\left[\mathrm{~d}(k)+\left(\left(\sigma_{1}^{2}(k)\right) / 2\right)\right]\right)\left(\sum_{k=1}^{N} \pi_{k}\left[\mathrm{~d}(k)+\gamma(k)+\mu(k)+\left(\left(\sigma_{2}^{2}(k)\right) / 2\right)\right]\right)}>1,
$$

then the solution $\left(S(t), I(t), R(t), \alpha_{t}\right)$ of model (1) has a unique stationary distribution, which means that the disease will persist.

Proof. In order to prove Theorem 2, we only need to check that the conditions in Lemma 1 are satisfied. First of all, we give some definitions of notations. Let $\check{a}:=\max _{i \in \mathcal{N}}\{a(i)\}$, $\widehat{a}:=\min _{i \in \mathcal{N}}\{a(i)\}$, and $a \vee b=\max \{a, b\}$. The first condition (A1) holds obviously from the assumption of Markov chain.

Let

$$
\mathscr{U}_{\varepsilon}=\left(\varepsilon, \frac{1}{\varepsilon}\right) \times\left(\varepsilon, \frac{1}{\varepsilon}\right) \times\left(\varepsilon, \frac{1}{\varepsilon}\right),
$$

where $\epsilon$ is a sufficiently small number. Thus, $\overline{\mathscr{U}_{\epsilon}} \subset \mathbb{R}_{+}^{3}$. From the expression of model (1), we can see that $\sigma(S, I, R, k)=$ $\operatorname{diag}\left(\sigma_{1}^{2}(k) S^{2}, \sigma_{2}^{2}(k) I^{2}, \sigma_{3}^{2}(k) R^{2}\right), k \in \mathcal{N}$, which implies that $\sigma(S, I, R, k)$ is positive definite and thus

$$
\lambda(k):=\min _{S, I, R \in \overline{\mathscr{U}_{e}}}\left\{\sigma_{1}^{2}(k) S^{2}, \sigma_{2}^{2}(k) I^{2}, \sigma_{3}^{2}(k) R^{2}\right\}>0 .
$$

We verify that condition (A2) holds. Now, we move to prove condition (A3). Some functions are defined as follows:

$$
\begin{aligned}
& V_{1}=-c_{1} \ln S-c_{2} \ln I+\frac{\check{a}}{\hat{\mu} q} I^{q}, \\
& V_{2}=-\ln S-\ln I, \\
& V_{3}=\frac{1}{p+1}(S+I+R)^{p+1},
\end{aligned}
$$

and the function $\tilde{V}=M\left(V_{1}+\omega_{k}\right)+V_{2}+V_{3}$, where the constants $c_{1}, c_{2}, p, M$, and $\omega_{k}$ will be specified later. Using the continuity of the function $\widetilde{V}$, we can take the minimum value of the function $\widetilde{V}\left(S_{*}, I_{*}, R_{*}, \omega_{*}\right)$ and let

$$
V(S, I, R, \omega):=\widetilde{V}(S, I, R, \omega)-\widetilde{V}\left(S_{*}, I_{*}, R_{*}, \omega_{*}\right) .
$$

In this way, the nonnegativity of the selected function $V$ can be guaranteed.

By making use of the Itô formula, we get that

Hence, for any $\xi \in \mathcal{U}$, it has $(\sigma(S, I, R, k) \xi, \xi) \geq \widehat{\lambda}|\xi|^{2}$.

$$
\begin{aligned}
\mathscr{L} V_{1}= & -\frac{c_{1}}{S}\left(\Lambda(k)-d(k) S-\frac{\beta(k) S I}{1+a(k) I^{q}}+\delta(k) R\right)+\frac{c_{1} \sigma_{1}^{2}(k)}{2}-\frac{c_{2}}{I}\left(\frac{\beta(k) S I}{1+a(k) I^{q}}-[d(k)+\gamma(k)+\mu(k)] I\right)+\frac{c_{2} \sigma_{2}^{2}(k)}{2} \\
& +\frac{\check{a}}{\widehat{\mu}}{ }^{q-1}\left(\frac{\beta(k) S I}{1+a(k) I^{q}}-[d(k)+\gamma(k)+\mu(k)] I\right)+\frac{\check{a}(q-1) \sigma_{2}^{2}(k) I^{q}}{2 \widehat{\mu}} \\
= & -\frac{c_{1} \Lambda(k)}{S}+c_{1} d(k)+\frac{c_{1} \beta(k) I}{1+a(k) I^{q}}-\frac{c_{1} \delta(k) R}{S}+\frac{c_{1} \sigma_{1}^{2}(k)}{2}-\frac{c_{2} \beta(k) S}{1+a(k) I^{q}}+c_{2}[d(k)+\gamma(k)+\mu(k)]+\frac{c_{2} \sigma_{2}^{2}(k)}{2}+1 \\
& +\frac{\check{a} \beta(k) I^{q} S}{\widehat{\mu}\left(1+a(k) I^{q}\right)}-\frac{\check{a}}{\widehat{\mu}} I^{q}[d(k)+\gamma(k)+\mu(k)]+\frac{\check{a}(q-1)}{2 \widehat{\mu}} \sigma_{2}^{2}(k) I^{q}-1 \\
\leq & -\frac{c_{1} \Lambda(k)}{S}-\frac{c_{2} \beta(k) S}{1+\check{a} I^{q}}-\check{a} I^{q}-1+c_{1}\left(d(k)+\frac{\sigma_{1}^{2}(k)}{2}\right)+\frac{c_{1} \beta(k) I^{1-q}}{a(k)}+c_{2}\left(d(k)+\gamma(k)+\mu(k)+\frac{\sigma_{2}^{2}(k)}{2}\right)+\frac{\check{a} \beta(k) S}{\widehat{\mu} a(k)}+1 \\
\leq & -3\left[c_{1} c_{2} \Lambda(k) \beta(k)\right]^{(1 / 3)}+c_{1}\left(d(k)+\frac{\sigma_{1}^{2}(k)}{2}\right)+1+c_{2}\left(d(k)+\gamma(k)+\mu(k)+\frac{\sigma_{2}^{2}(k)}{2}\right)+\frac{c_{1} \check{\beta}}{\widehat{a}} I^{1-q}+\frac{\check{a} \check{\beta}}{\widehat{\mu} \widehat{a}} S .
\end{aligned}
$$


Let $A(k)=-3\left[c_{1} c_{2} \Lambda(k) \beta(k)\right]^{(1 / 3)}+c_{1}\left(\mathrm{~d}(k)+\left(\left(\sigma_{1}^{2}(k)\right)\right.\right.$

$/ 2))+c_{2}\left(d(k)+\gamma(k)+\mu(k)+\left(\left(\sigma_{2}^{2}(k)\right) / 2\right)\right)$, and select

$$
\left(c_{1}\right)^{(1 / 3)}=\frac{\sum_{k=1}^{N} \pi_{k}[\Lambda(k) \beta(k)]^{(1 / 3)}}{\left(\sum_{k=1}^{N} \pi_{k}\left[\mathrm{~d}(k)+\left(\left(\sigma_{1}^{2}(k)\right) / 2\right)\right]\right)^{(2 / 3)}\left(\sum_{k=1}^{N} \pi_{k}\left[\mathrm{~d}(k)+\gamma(k)+\mu(k)+\left(\left(\sigma_{2}^{2}(k)\right) / 2\right)\right]\right)^{(1 / 3)}},
$$

as well as

$$
\left(c_{2}\right)^{(1 / 3)}=\frac{\sum_{k=1}^{N} \pi_{k}[\Lambda(k) \beta(k)]^{(1 / 3)}}{\left(\sum_{k=1}^{N} \pi_{k}\left[d(k)+\left(\left(\sigma_{1}^{2}(k)\right) / 2\right)\right]\right)^{(1 / 3)}\left(\sum_{k=1}^{N} \pi_{k}\left[\mathrm{~d}(k)+\gamma(k)+\mu(k)+\left(\left(\sigma_{2}^{2}(k)\right) / 2\right)\right]\right)^{(2 / 3)}} .
$$

Consider the system

$$
\Gamma \omega=\sum_{k=1}^{N} \pi_{k} A(k)(1,1, \ldots, 1)^{T}-A
$$

where $A=(A(1), A(2), \ldots, A(N))^{T}$. Hence, it has the solution $\omega=(\omega(1), \omega(2), \ldots, \omega(N))^{T}$ such that $\sum_{j=1}^{N} \gamma_{k j} \omega_{j}+$ $A(k)=\sum_{k=1}^{N} \pi_{k} A(k)$. Therefore, we obtain that

$$
\begin{aligned}
\mathscr{L}\left(V_{1}+\omega_{k}\right) & \leq A_{k}+1+\frac{c_{1} \check{\beta}}{\widehat{a}} I^{1-q}+\frac{\check{a} \check{\beta}}{\widehat{\mu} \widehat{a}} S+\sum_{j=1}^{N} \gamma_{k j} \omega_{j} \\
& \leq-\lambda+\frac{c_{1} \check{\beta}}{\widehat{a}} I^{1-q}+\frac{\check{a} \check{\beta}}{\widehat{\mu} \widehat{a}} S .
\end{aligned}
$$

Here, $\lambda:=R_{1}^{S}-1$ and $R_{1}^{S}$ is defined in (7).

$$
\begin{aligned}
& \mathscr{L}(-\ln S)=-\frac{\Lambda(k)}{S}+d(k)+\frac{\beta(k) I}{1+a(k) I^{q}}-\frac{\delta(k) R}{S}+\frac{\sigma_{1}^{2}(k)}{2}, \\
& \mathscr{L}(-\ln R)=-\frac{\gamma(k) I}{R}+d(k)+\delta(k)+\frac{\sigma_{3}^{2}(k)}{2}, \\
& \mathscr{L} V_{3}=(S+I+R)^{p}[\Lambda(k)-\mathrm{d}(k)(S+I+R)-\mu(k) I]+p(S+I+R)^{p-1}\left(\frac{\sigma_{1}^{2}(k)}{2} S^{2}+\frac{\sigma_{2}^{2}(k)}{2} I^{2}+\frac{\sigma_{1}^{2}(k)}{2} R^{2}\right), \\
& \leq \check{\Lambda}(S+I+R)^{p}-\left(\widehat{d}-\frac{p}{2}\left(\check{\sigma}_{1}^{2}+\check{\sigma}_{2}^{2}+\check{\sigma}_{3}^{2}\right)\right)(S+I+R)^{p+1} \\
& \leq \sup _{(S, I, R) \in \mathbb{R}_{+}^{3}}\left\{\check{\Lambda}(S+I+R)^{p}-\frac{\widehat{d}-(p / 2)\left(\check{\sigma}_{1}^{2}+\check{\sigma}_{2}^{2}+\check{\sigma}_{3}^{2}\right)}{2}(S+I+R)^{p+1}\right\}-\frac{\widehat{d}-(p / 2)\left(\check{\sigma}_{1}^{2}+\check{\sigma}_{2}^{2}+\check{\sigma}_{3}^{2}\right)}{2}(S+I+R)^{p+1} \\
& =: E-\frac{\widehat{d}-(p / 2)\left(\check{\sigma}_{1}^{2}+\check{\sigma}_{2}^{2}+\check{\sigma}_{3}^{2}\right)}{2}(S+I+R)^{p+1} \text {. }
\end{aligned}
$$

Again, we apply the Itô formula to $-\ln S,-\ln I$, and $V_{3}$ to obtain 
Here, we choose sufficiently small constant $p$ such that

$$
\widehat{d}-\frac{p}{2}\left(\check{\sigma}_{1}^{2}+\check{\sigma}_{2}^{2}+\check{\sigma}_{3}^{2}\right)>0 \text {. }
$$

Thus, $E<\infty$ and

$\mathscr{L} V_{3} \leq E-\frac{\widehat{d}-(p / 2)\left(\check{\sigma}_{1}^{2}+\check{\sigma}_{2}^{2}+\check{\sigma}_{3}^{2}\right)}{2}\left(S^{p+1}+I^{p+1}+R^{p+1}\right)$.

Consequently,

$$
\begin{aligned}
\mathscr{L} V \leq & -M \lambda+\left(M c_{1}+1\right) \frac{\check{\beta}}{\widehat{a}} I^{1-q}+\frac{M \check{a} \check{\beta}}{\widehat{\mu} \widehat{a}} S-\frac{\widehat{\Lambda}}{S}-\frac{\widehat{\delta} R}{S}-\frac{\widehat{\gamma} I}{R} \\
& +2 \check{d}+\check{\delta}+E \\
& +\frac{\check{\sigma}_{1}^{2}+\check{\sigma}_{3}^{2}}{2}-\frac{\widehat{d}-(p / 2)\left(\check{\sigma}_{1}^{2}+\check{\sigma}_{2}^{2}+\check{\sigma}_{3}^{2}\right)}{2} \\
& \cdot\left(S^{p+1}+I^{p+1}+R^{p+1}\right) .
\end{aligned}
$$

Select sufficiently large $M$ and sufficiently small $\varrho$ such that the following formulas hold:

$$
\begin{array}{r}
-\frac{\widehat{\Lambda}}{\varrho}+F \leq-1, \\
-M \lambda+\left(M c_{1}+1\right) \frac{\check{\beta}}{\widehat{a}}{ }^{1-q}+G \leq-1, \\
-\frac{\widehat{\gamma}}{\varrho}+H \leq-1, \\
-M \lambda-\frac{\widehat{d}-(p / 2)\left(\check{\sigma}_{1}^{2}+\check{\sigma}_{2}^{2}+\check{\sigma}_{3}^{2}\right)}{4}\left(\frac{1}{\varrho}\right)^{p+1}+K \leq-1,
\end{array}
$$

where the constants $F, G, H$, and $K$ are all finite and their representations can been seen in (27)-(29) and (31).

For the constant $\varrho$ selected above, define a set

$$
\mathscr{D}_{\varrho}=\left\{(S, I, R) \in \mathbb{R}_{+}^{3}: \varrho \leq S \leq \frac{1}{\varrho}, \varrho \leq I \leq \frac{1}{\varrho}, \varrho^{2} \leq R \leq \frac{1}{\varrho^{2}}\right\} \text {. }
$$

Hence, we can divide the set $\mathbb{R}_{+}^{3} \backslash \mathscr{D}_{\mathrm{Q}}$ into six regions

$$
\begin{aligned}
& \mathscr{D}_{1}=\left\{(S, I, R) \in \mathbb{R}_{+}^{3}: 0<S<\varrho\right\}, \\
& \mathscr{D}_{2}=\left\{(S, I, R) \in \mathbb{R}_{+}^{3}: 0<I<\varrho\right\}, \\
& \mathscr{D}_{3}=\left\{(S, I, R) \in \mathbb{R}_{+}^{3}: 0<R<\varrho^{2}, I \geq \varrho\right\}, \\
& \mathscr{D}_{4}=\left\{(S, I, R) \in \mathbb{R}_{+}^{3}: S>\frac{1}{\varrho}\right\}, \\
& \mathscr{D}_{5}=\left\{(S, I, R) \in \mathbb{R}_{+}^{3}: I>\frac{1}{\varrho}\right\}, \\
& \mathscr{D}_{6}=\left\{(S, I, R) \in \mathbb{R}_{+}^{3}: R>\frac{1}{\varrho^{2}}\right\} .
\end{aligned}
$$

In what follows, we will prove that $\mathscr{L V} \leq-1$ in $\mathbb{R}_{+}^{3} \backslash \mathscr{D}_{\varrho}$, that is, $\mathscr{L} V \leq-1$ in the regions $\mathscr{D}_{i}, i=1, \ldots, 6$.

Case 1: when $(S, I, R) \in \mathscr{D}_{1}$, it has

$$
\mathscr{L} V \leq-\frac{\widehat{\Lambda}}{\varrho}+F
$$

where $F=\sup _{(S, I, R) \in \mathbb{R}_{+}^{3}}\left\{-M \lambda+\left(M c_{1}+1\right)(\check{\beta} / \widehat{a}) I^{1-q}+\right.$ $((M \check{a} \check{\beta}) / \hat{\mu} \widehat{a}) S+2 \check{d}+\check{\delta}+E+\left(\left(\check{\sigma}_{1}^{2}+\check{\sigma}_{3}^{2}\right) / 2\right)-((\widehat{d}-(p / 2)$ $\left.\left.\left.\left(\check{\sigma}_{1}^{2}+\check{\sigma}_{2}^{2}+\check{\sigma}_{3}^{2}\right)\right) / 2\right)\left(S^{p+1}+I^{p+1}+R^{p+1}\right)\right\}<\infty$.

From the result of (21), one has $\mathscr{L} \mathrm{V} \leq-1$.

Case 2: when $(S, I, R) \in \mathscr{D}_{2}$, it has

$$
\mathscr{L} V \leq-M \lambda+\left(M c_{1}+1\right) \frac{\check{\beta}}{\widehat{a}}(\varrho)^{1-q}+G,
$$

where $G=\sup _{(S, I, R) \in \mathbb{R}_{+}^{3}}\{((M \check{a} \check{\beta}) /(\widehat{\mu} \widehat{a})) S+2 \check{d}+\check{\delta}+E+$ $\left(\left(\check{\sigma}_{1}^{2}+\check{\sigma}_{3}^{2}\right) / 2\right)-\left(\left(\widehat{d}-(p / 2)\left(\check{\sigma}_{1}^{2}+\check{\sigma}_{2}^{2}+\check{\sigma}_{3}^{2}\right)\right) / 2\right)\left(S^{p+1}+\right.$ $\left.\left.I^{p+1}+R^{p+1}\right)\right\}<\infty$.

$\mathscr{L} V \leq-1$ can be obtained by (22).

Case 3: when $(S, I, R) \in \mathscr{D}_{3}$, we have

$$
\mathscr{L} V \leq-\frac{\widehat{\gamma} I}{R}+H \leq-\frac{\widehat{\gamma}}{\varrho}+H .
$$

Here, $H=\sup _{(S, I, R) \in \mathbb{R}_{+}^{3}}\left\{-M \lambda+\left(M c_{1}+1\right)(\check{\beta} / \widehat{a}) I^{1-q}+\right.$ $((M \check{a} \check{\beta}) / \hat{\mu} \widehat{a}) S+2 \check{d}+\check{\delta}+E+\left(\left(\check{\sigma}_{1}^{2}+\check{\sigma}_{3}^{2}\right) / 2\right)-((\widehat{d}-(p / 2)$ $\left.\left.\left.\left(\check{\sigma}_{1}^{2}+\check{\sigma}_{2}^{2}+\check{\sigma}_{3}^{2}\right)\right) / 2\right)\left(S^{p+1}+I^{p+1}+R^{p+1}\right)\right\}<\infty$.

$\mathscr{L} V \leq-1$ can be obtained from the result of (23).

Case 4: when $(S, I, R) \in \mathscr{D}_{4}$, we have

$$
\begin{aligned}
\mathscr{L} V & \leq-M \lambda-\frac{\widehat{d}-(p / 2)\left(\check{\sigma}_{1}^{2}+\check{\sigma}_{2}^{2}+\check{\sigma}_{3}^{2}\right)}{4} S^{p+1}+K, \\
& \leq-M \lambda-\frac{\widehat{d}-(p / 2)\left(\check{\sigma}_{1}^{2}+\check{\sigma}_{2}^{2}+\check{\sigma}_{3}^{2}\right)}{4}\left(\frac{1}{\varrho}\right)^{p+1}+K,
\end{aligned}
$$

where

$$
\begin{aligned}
K= & \sup _{(S, I, R) \in \mathbb{R}_{+}^{3}}\left\{\left(M c_{1}+1\right) \frac{\check{\beta}}{\widehat{a}} I^{1-q}+\frac{M \check{a} \check{\beta}}{\widehat{\mu} \widehat{a}} S+2 \check{d}+\check{\delta}\right. \\
& +E+\frac{\check{\sigma}_{1}^{2}+\check{\sigma}_{3}^{2}}{2}-\frac{\widehat{d}-(p / 2)\left(\check{\sigma}_{1}^{2}+\check{\sigma}_{2}^{2}+\check{\sigma}_{3}^{2}\right)}{4} \\
& \left.\cdot\left(S^{p+1}+I^{p+1}+R^{p+1}\right)\right\}<\infty .
\end{aligned}
$$

Thus, we can get that $\mathscr{L V} \leq-1$ with the help of (24). Similarly, $\quad(S, I, R) \in \mathscr{D}_{5}$ and $(S, I, R) \in \mathscr{D}_{6}$ can be proved in a similar way. So far, we have proved that $\mathscr{L} V \leq-1$ in region $\mathbb{R}_{+}^{3} \backslash \mathscr{D}_{\varrho}$. By virtue of Lemma 1 , the positive recurrence of model (1) can be obtained. 
Remark 1. We can see from the proof that in order to obtain the positive recurrence of the model, different Lyapunov functions are constructed to reach the goal.

\section{Extinction of Disease}

In this section, we will discuss the conditions for extinction of disease in model (1). Let us start with a lemma as follows.

Lemma 2. Assume that there exists some constant $p>1$ such that $\widehat{d}-((p-1) / 2)\left(\check{\sigma}_{1}^{2} \vee \check{\sigma}_{2}^{2} \vee \check{\sigma}_{3}^{2}\right)>0$ and $(S(t), I(t), R(t)$, $\left.\alpha_{t}\right)$ is the solution of model (1) with the initial value $\left(S(t), I(t), R(t), \alpha_{0}\right)$, then

$$
\lim _{t \rightarrow \infty} \frac{S(t)+I(t)+R(t)}{t}=0, \quad \text { a.s. }
$$

By the nonnegative property of $S(t), I(t), R(t)$, it has

$$
\begin{aligned}
& \lim _{t \rightarrow \infty} \frac{S(t)}{t}=0, \\
& \lim _{t \rightarrow \infty} \frac{I(t)}{t}=0, \\
& \lim _{t \rightarrow \infty} \frac{R(t)}{t}=0, \quad \text { a.s. }
\end{aligned}
$$

In addition,

$$
\begin{aligned}
& \lim _{t \rightarrow \infty} \frac{\int_{0}^{t} \sigma_{1}\left(\alpha_{s}\right) S(s) \mathrm{d} B_{1}(s)}{t}=0, \\
& \lim _{t \rightarrow \infty} \frac{\int_{0}^{t} \sigma_{2}\left(\alpha_{s}\right) I(s) \mathrm{d} B_{2}(s)}{t}=0, \\
& \lim _{t \rightarrow \infty} \frac{\int_{0}^{t} \sigma_{3}\left(\alpha_{s}\right) R(s) \mathrm{d} B_{3}(s)}{t}=0, \quad \text { a.s. }
\end{aligned}
$$

The proof is similar to that in [28]; we omit it here.

Theorem 3. Let $m:=\max _{i \in \mathcal{N}}\{\check{\delta} / d(i)+\delta(i)\} \quad$ and $c:=\max _{i \in \mathcal{N}}\{(\beta(i)) / d(i)\}$. If $(d(i)+\gamma(i)+\mu(i))-m \gamma(i) \geq 0$ for each $i \in \mathcal{N}$, then

$$
\limsup _{t \rightarrow \infty} \frac{\ln I(t)}{t} \leq \sum_{i=1}^{N} \pi_{i}\left[c \Lambda(i)-\left(\mathrm{d}(i)+\gamma(i)+\mu(i)+\frac{\sigma_{2}^{2}(i)}{2}\right)\right], \quad \text { a.s. }
$$

Assume further that

$$
R_{0}^{S}:=\frac{c \sum_{i=1}^{N} \pi_{i} \Lambda(i)}{\sum_{i=1}^{N} \pi_{i}\left(d(i)+\gamma(i)+\mu(i)+\left(\sigma_{2}^{2}(i) / 2\right)\right)}<1 .
$$

Then, the disease will extinct exponentially almost surely Proof. Applying Itô formula to $\ln I(t)$ yields

$$
\begin{aligned}
\mathrm{d} \ln I(t) & =\left[\frac{\beta\left(\alpha_{t}\right) S}{1+a\left(\alpha_{t}\right) I^{q}}-\left(d\left(\alpha_{t}\right)+\gamma\left(\alpha_{t}\right)+\mu\left(\alpha_{t}\right)+\frac{\sigma_{2}^{2}\left(\alpha_{t}\right)}{2}\right)\right] \mathrm{d} t+\sigma_{2}\left(\alpha_{t}\right) \mathrm{d} B_{2}(t), \\
& \leq\left[\beta\left(\alpha_{t}\right) S-\left(\mathrm{d}\left(\alpha_{t}\right)+\gamma\left(\alpha_{t}\right)+\mu\left(\alpha_{t}\right)+\frac{\sigma_{2}^{2}\left(\alpha_{t}\right)}{2}\right)\right] \mathrm{d} t+\sigma_{2}\left(\alpha_{t}\right) \mathrm{d} B_{2}(t) .
\end{aligned}
$$

Add the first two equations in (1) to get

$$
\begin{aligned}
\mathrm{d}(S+I) & =\left(\Lambda\left(\alpha_{t}\right)-\mathrm{d}\left(\alpha_{t}\right) S+\delta\left(\alpha_{t}\right) R-\left(d\left(\alpha_{t}\right)+\gamma\left(\alpha_{t}\right)+\mu\left(\alpha_{t}\right)\right) I\right) \mathrm{d} t+\sigma_{1}\left(\alpha_{t}\right) S \mathrm{~d} B_{1}(t)+\sigma_{2}\left(\alpha_{t}\right) I \mathrm{~d} B_{2}(t) \\
& \leq\left(\Lambda\left(\alpha_{t}\right)-d\left(\alpha_{t}\right) S+\check{\delta} R-\left(d\left(\alpha_{t}\right)+\gamma\left(\alpha_{t}\right)+\mu\left(\alpha_{t}\right)\right) I\right) \mathrm{d} t+\sigma_{1}\left(\alpha_{t}\right) S \mathrm{~d} B_{1}(t)+\sigma_{2}\left(\alpha_{t}\right) I \mathrm{~d} B_{2}(t) .
\end{aligned}
$$

This, along with the third equation in (1), yields

$$
\begin{aligned}
\mathrm{d}(S+I+m R) & \leq\left(\Lambda\left(\alpha_{t}\right)-d\left(\alpha_{t}\right) S+\left[\check{\delta}-m\left(\mathrm{~d}\left(\alpha_{t}\right)+\delta\left(\alpha_{t}\right)\right)\right] R-\left(d\left(\alpha_{t}\right)+\gamma\left(\alpha_{t}\right)+\mu\left(\alpha_{t}\right)-m \gamma\left(\alpha_{t}\right)\right) I\right) \mathrm{d} t \\
& +\sigma_{1}\left(\alpha_{t}\right) S(t) \mathrm{d} B_{1}(t)+\sigma_{2}\left(\alpha_{t}\right) I(t) \mathrm{d} B_{2}(t)+m \sigma_{3}\left(\alpha_{t}\right) R(t) \mathrm{d} B_{3}(t) \\
& \leq\left(\Lambda\left(\alpha_{t}\right)-d\left(\alpha_{t}\right) S\right) \mathrm{d} t+\sigma_{1}\left(\alpha_{t}\right) S(t) \mathrm{d} B_{1}(t)+\sigma_{2}\left(\alpha_{t}\right) I(t) \mathrm{d} B_{2}(t)+m \sigma_{3}\left(\alpha_{t}\right) R(t) \mathrm{d} B_{3}(t) .
\end{aligned}
$$




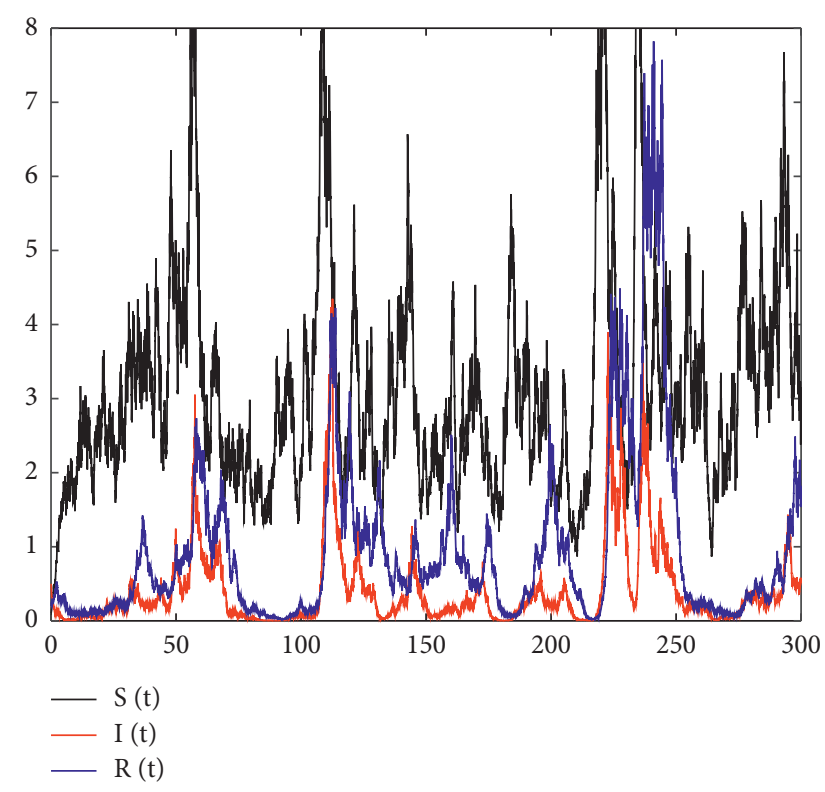

FIGURE 1: The trajectory of SIRS model 1 with values of parameters in example 1 and initial value $S(0)=0.1, I(0)=0.5$, and $R(0)=0.2$.

Integrating (24) and $c d(S+I+m R)$ from 0 to $t$ and dividing by $t$ for both sides of formula, one has

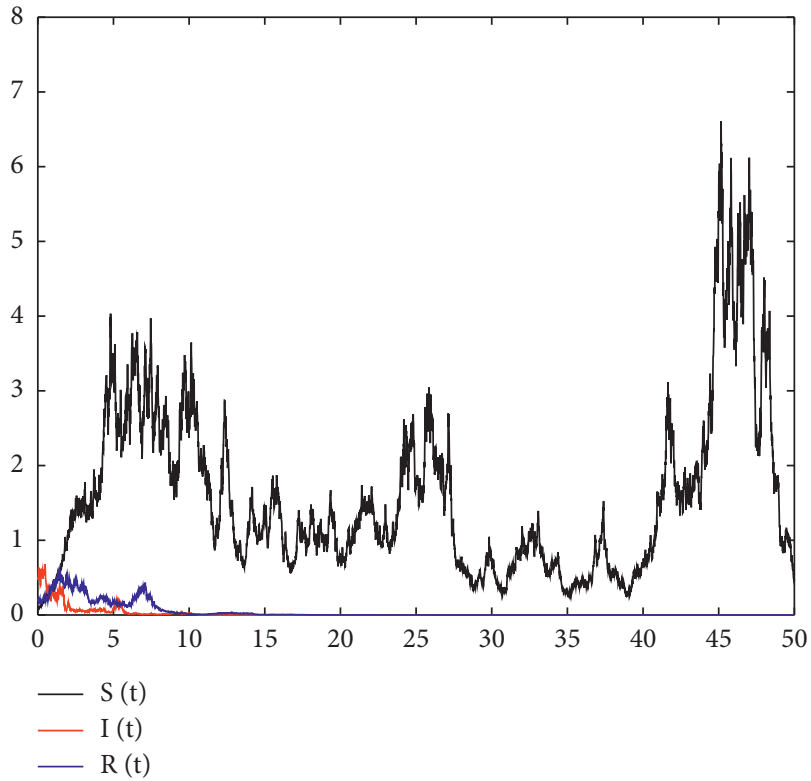

FIGURE 2: The trajectory of SIRS model 1 with values of parameters in example 2 and initial value $S(0)=0.1, I(0)=0.5$, and $R(0)=0.2$.

$$
\begin{aligned}
\frac{\ln I(t)}{t} \leq & \frac{\ln I(0)}{t}+\frac{1}{t} \int_{0}^{t} c \Lambda\left(\alpha_{s}\right) \mathrm{d} s+\frac{1}{t} \int_{0}^{t}\left(\beta\left(\alpha_{s}\right)-c d\left(\alpha_{s}\right)\right) S(s) \mathrm{d} s \\
& -\frac{1}{t} \int_{0}^{t}\left(\mathrm{~d}\left(\alpha_{s}\right)+\gamma\left(\alpha_{s}\right)+\mu\left(\alpha_{s}\right)+\frac{\sigma_{2}^{2}\left(\alpha_{s}\right)}{2}\right) \mathrm{d} s-\frac{c(S(t)+I(t)+m R(t))}{t}+\frac{c(S(0)+I(0)+m R(0))}{t} \\
& +\frac{1}{t} \int_{0}^{t} c \sigma_{1}\left(\alpha_{s}\right) S(s) \mathrm{d} B_{1}(s)+\frac{1}{t} \int_{0}^{t} c \sigma_{2}\left(\alpha_{s}\right) I(s) \mathrm{d} B_{2}(s)+\frac{1}{t} \int_{0}^{t} m c \sigma_{3}\left(\alpha_{s}\right) R(s) \mathrm{d} B_{3}(s)+\frac{1}{t} \int_{0}^{t} \sigma_{2}\left(\alpha_{s}\right) \mathrm{d} B_{2}(s) .
\end{aligned}
$$

By virtue of Lemma 2 and ergodicity of Markov chain, (35) can be obtained. If $R_{0}^{S}<1$, then $((\ln I(t)) / t)<0$, which means that the disease will extinct exponentially. The proof is completed.

\section{Examples and Simulations}

In this section, some examples are constructed and their simulations are presented to verify the abovementioned results.

Let $\left(\alpha_{t}\right)_{t \geq 0}$ be the Markov chain with state space $\mathcal{N}=$ $\{1,2\}$ and its generator is $Q_{1}=\left(\begin{array}{cc}-m & m \\ n & -n\end{array}\right)$. Thus, its stationary distribution is $\pi=\left(\pi_{1}, \pi_{2}\right)=((n /(m+n))$, $(m /(m+n)))$.

Example 1. We will give the values of the abovementioned parameters in the two states in the form of vectors, that is, $a=[3,1]$ means $a(1)=3, a(2)=1$ in two states. To verify the result of Theorem 2 , we assume that $m=1, n=2$, $q=0.5, \quad a=[2,1], \quad \Lambda=[0.4,0.35], \quad d=[0.1,0.08]$, $\beta=[0.5,0.4], \mu=[0.1,0.05], \gamma=[0.5,0.7], \delta=[0.1,0.3]$, $\sigma_{1}=[0.2,0.4], \sigma_{2}=[0.4,0.1]$, and $\sigma_{1}=[0.3,0.2]$ such that the threshold in Theorem $2, R_{1}^{S}=1.6761>1$. According to the result of the Theorem 2 , the model has unique stationary distribution and the disease will persist, see Figure 1.

Example 2. To verify the result of Theorem 3, let $m=1$, $n=2, \quad q=0.5, \quad a=[2,1], \quad \Lambda=[0.2,0.3], \quad d=[0.1,0.08]$, $\beta=[0.2,0.3], \mu=[0.1,0.05], \gamma=[0.5,0.35], \delta=[0.2,0.4]$, $\sigma_{1}=[0.5,0.7], \sigma_{2}=[1,0.3]$, and $\sigma_{1}=[0.4,1]$ such that the threshold in Theorem $3, R_{0}^{S}=0.8974<1$. According to the result of Theorem 3, the disease will die out, see Figure 2.

\section{Conclusions}

In this paper, the dynamic behavior of a class of hybrid SIRS model with nonlinear incidence is studied. Firstly, the condition under which the positive recurrence exists is provided, and then we give the threshold $R_{0}^{S}$ for disease extinction, that 
is, when $R_{0}^{S}<1$, the disease will die out. Finally, some examples are constructed to verify the conclusion.

Some other issues deserve further study. For example, the incidence function can be more generalized, or Le vy noise can be added to make the model more realistic. We can also investigate other properties of the model, such as stability and so on. These are left to study in the future.

\section{Data Availability}

The data used to support the findings of this study are included within the article.

\section{Conflicts of Interest}

The authors declare that they have no conflicts of interest.

\section{Acknowledgments}

This work was supported by the National Natural Science Foundation of China (no. 11661065) and the Scientific Research Fund of the Jiangxi Provincial Education Department (no. GJJ191145).

\section{References}

[1] A. Settati, A. Lahrouz, M. El Jarroudi, and M. Jarroudi, "Dynamics of hybrid switching diffusions SIRS model," Journal of Applied Mathematics and Computing, vol. 52, no. 12, pp. 101-123, 2016.

[2] D. Li, S. Liu, and J. A. Cui, "Threshold dynamics and ergodicity of an SIRS epidemic model with semi-Markov switching," Journal of Differential Equations, vol. 266, no. 7, pp. 3973-4017, 2019.

[3] X. Meng, S. Zhao, T. Feng, and T. Zhang, "Dynamics of a novel nonlinear stochastic SIS epidemic model with double epidemic hypothesis," Journal of Mathematical Analysis and Applications, vol. 433, no. 1, pp. 227-242, 2016.

[4] D. Li, S. Liu, and J. A. Cui, "Threshold dynamics and ergodicity of an SIRS epidemic model with Markovian switching," Journal of Differential Equations, vol. 263, no. 12, pp. 88738915, 2017.

[5] Q. Liu, D. Jiang, N. Shi, T. Hayat, and A. Alsaedi, "Stationary distribution and extinction of a stochastic SIRS epidemic model with standard incidence," Physica A: Statistical Mechanics and Its Applications, vol. 469, pp. 510-517, 2017.

[6] Y. Cai, K. Yun, and W. Wang, "A stochastic sirs epidemic model with nonlinear incidence rate," Applied Mathematics and Computation, vol. 305, pp. 221-240, 2017.

[7] G. Hussain, A. Khan, M. Zahri, and G. Zaman, "Stochastic permanence of an epidemic model with a saturated incidence rate," Chaos, Solitons and Fractals, vol. 139, no. 7, Article ID 110005, 2020.

[8] X. Guo and J. Luo, "Stationary distribution and extinction of SIR model with nonlinear incident rate under Markovian switching," Physica A: Statistical Mechanics and Its Applications, vol. 505, pp. 471-481, 2018.

[9] Q. Liu, D. Jiang, T. Hayat, and B. Ahmad, "Stationary distribution and extinction of a stochastic SIRI epidemic model with relapse," Stochastic Analysis and Applications, vol. 36, no. 1, pp. 138-151, 2018.

[10] Y. Zhang, K. Fan, S. Gao, and S. Chen, "A remark on stationary distribution of a stochastic SIR epidemic model with double saturated rates," Applied Mathematics Letters, vol. 76, pp. 46-52, 2018.

[11] X. Zhang, D. Jiang, A. Alsaedi, and T. Hayat, "Stationary distribution of stochastic SIS epidemic model with vaccination under regime switching," Applied Mathematics Letters, vol. 59, pp. 87-93, 2016.

[12] Q. Liu and D. Q. Jiang, "Stationary distribution of a stochastic SIS epidemic model with double diseases and the BeddingtonDeAngelis incidence," Chaos, vol. 27, no. 083126, p. 8, 2017.

[13] A. Lahrouz and A. Settati, "Necessary and sufficient condition for extinction and persistence of SIRS system with random perturbation," Applied Mathematics and Computation, vol. 233, pp. 10-19, 2014.

[14] E. Tornatore, S. Maria Buccellato, and P. Vetro, "Stability of a stochastic SIR system," Physica A: Statistical Mechanics and Its Applications, vol. 354, pp. 111-126, 2005.

[15] M. Lu, J. Huang, S. Ruan, and P. Yu, "Bifurcation analysis of an SIRS epidemic model with a generalized nonmonotone and saturated incidence rate," Journal of Differential Equations, vol. 267, no. 3, pp. 1859-1898, 2019.

[16] P. E. Kloeden and C. Pötzsche, "Nonautonomous bifurcation scenarios in SIR models," Mathematical Methods in the Applied Sciences, vol. 38, no. 16, pp. 3495-3518, 2015.

[17] H. Laarabi, E. H. Labriji, M. Rachik, and A. Kaddar, "Optimal control of an epidemic model with a saturated incidence rate," Nonlinear Analysis: Modelling and Control, vol. 17, no. 4, pp. 448-459, 2012.

[18] W.-m. Liu, S. A. Levin, and Y. Iwasa, "Influence of nonlinear incidence rates upon the behavior of SIRS epidemiological models," Journal of Mathematical Biology, vol. 23, no. 2, pp. 187-204, 1986.

[19] F. Wei and R. Xue, "Stability and extinction of SEIR epidemic models with generalized nonlinear incidence," Mathematics and Computers in Simulation, vol. 170, pp. 1-15, 2020.

[20] N. Dieu, T. Fugo, and N. H. Du, "Asymptotic behaviors of stochastic epidemic models with jump-diffusion," Applied Mathematical Modelling, vol. 86, pp. 259-270, 2020.

[21] T. Caraballo, A. Settati, M. E. Fatini, A. Lahrouz, and A. Imlahi, "Global stability and positive recurrence of a stochastic SIS model with Lévy noise perturbation Levy noise perturbation," Physica A: Statistical Mechanics and Its Applications, vol. 523, pp. 677-690, 2019.

[22] G. Lan, Z. Lin, C. Wei, and S. Zhang, "A stochastic SIRS epidemic model with non-monotone incidence rate under regime-switching," Journal of the Franklin Institute, vol. 356, no. 16, pp. 9844-9866, 2019.

[23] N. T. Du, N. H. Du, P. Auger, and N. H. Dang, "Dynamical behavior of a stochastic SIRS epidemic model," Mathematical Modelling of Natural Phenomena, vol. 10, no. 2, pp. 56-73, 2014.

[24] J. H. Bao and J. H. Shao, "Asymptotic behavior of SIRS Models in State-dependent Random Environments," Nonlinear Analysis-Hybrid Systems Nonlinear Anal-Hybri., vol. 38, Article ID 100914, 18 pages, 2020.

[25] A. Economou and M. J. Lopez-Herrero, "The deterministic SIS epidemic model in a Markovian random environment," Journal of Mathematical Biology, vol. 73, no. 1, pp. 91-121, 2016.

[26] Q. Liu, D. Jiang, T. Hayat, A. Alsaedi, and B. Ahmad, "Dynamics of a multigroup SIQS epidemic model under regime switching," Stochastic Analysis and Applications, vol. 38, no. 5, pp. 769-796, 2020.

[27] R. Khasminskii, Stochastic Stability of Differential Equations, Berlin, Heidelberg, 2012.

[28] Y. Zhao and D. Jiang, "The threshold of a stochastic SIS epidemic model with vaccination," Applied Mathematics and Computation, vol. 243, pp. 718-727, 2014. 